\title{
Introducing Regina, The 3-Manifold Topology Software
}

\author{
Benjamin A. Burton
}

\section{CONTENTS}

1. Introduction

2. Features

3. Applications

Acknowledgments

References

2000 AMS Subject Classification: Primary 57M04, 57N10; Secondary 57M27

Keywords: Triangulation, software, normal surface, angle structure, census
An overview is presented of Regina, a freely available software package for 3-manifold topologists. In addition to working with 3-manifold triangulations, Regina includes support for normal surfaces and angle structures. The features of the software are described in detail, followed by examples of research projects in which Regina has been used.

\section{INTRODUCTION}

Experimental work in the study of 3-manifold topology has been a historically challenging task. Topological calculations for even simple 3-manifold triangulations are often remarkably difficult to perform by hand. Furthermore, triangulations and 3-manifolds are difficult to represent and manipulate using standard programming languages. Because of this, relatively little software has been available until recently to assist with these calculations.

One prominent exception is SnapPea [Weeks 91], under development for over a decade, which provides excellent support for the study of hyperbolic 3-manifolds. More recently a number of other tools have become available, many of which are listed at the computational topology web site http://www.computop.org/.

Regina is a software package that unites a number of standard 3-manifold topology algorithms and procedures within a friendly user interface, as well as adding previously unavailable features to the current body of experimental tools. Under development since 1999, its growth has to a large extent been guided by its use in a variety of research projects, some of which are noted in Section 3. Algorithms that are implemented include triangulation simplification, normal surface enumeration, angle structure analysis and the calculation of algebraic and combinatorial invariants.

This software is released under the GNU General Public License and is publicly available from http://regina.

(c) A K Peters, Ltd. $1058-6458 / 2004 \$ 0.50$ per page Experimental Mathematics 13:3, page 267 
sourceforge.net/. Both the user interface and the underlying programmer's interface are thoroughly documented.

Regina continues to grow and currently enjoys a new release every few months. Special thanks must go to David Letscher who assisted with the early phases of development. Thanks also to Marc Culler, Nathan Dunfield, William Jaco, Richard Rannard, J. Hyam Rubinstein, and Jeff Weeks for many fruitful discussions.

In Section 2 we present a detailed description of the capabilities of Regina. Section 3 closes with some examples of research projects that have made use of this software.

\section{FEATURES}

Since its inception, Regina has been carefully designed for rigour and extensibility. The software is written primarily in the $\mathrm{C}++$ programming language and runs under GNU/Linux and related operating systems. A list of the more noteworthy features of Regina is presented below.

\subsection{Triangulations}

The primary objects with which a user interacts when running Regina are 3-manifold triangulations. As such, a large part of the software is devoted to the creation, analysis, and manipulation of triangulations.

2.1.1 Creation. The following methods are supported for creating triangulations:

- manual construction of triangulations by entering individual tetrahedron face identifications by hand;

- automatic generation of standard triangulations such as layered solid tori and layered lens spaces [Jaco and Rubinstein 02, Jaco and Rubinstein 03b];

- automatic construction of Seifert fibred spaces over the 2-sphere with up to three exceptional fibres;

- reconstruction of triangulations from dehydration strings [Callahan et al. 99];

- importing triangulations saved from SnapPea [Weeks 91].

2.1.2 Analysis. Properties of a triangulation that the software can compute include the following:

- detailed combinatorial information about the skeleton and boundary components, including vertex links and the shapes formed by the various triangulation faces;
- a variety of homology and homotopy groups;

- the quantum invariants of Turaev and Viro [Turaev and Viro 92];

- 3-sphere recognition, as well as a complete connected sum decomposition for closed orientable triangulations [Jaco and Rubinstein 02];

- triangulation attributes relating to the existence of particular types of normal surface, such as 0efficiency [Jaco and Rubinstein 02] and the existence of splitting surfaces (described in Section 2.3.3).

Pairs of triangulations can be tested for direct isomorphism, or for whether one triangulation is isomorphic to a subcomplex of another. In addition, the software contains a variety of recognition routines for detecting particular well-formed structures within a triangulation. These routines recognise smaller building blocks that often appear within larger triangulations, such as layered solid tori [Jaco and Rubinstein 02, Jaco and Rubinstein 03b] and thin $I$-bundles [Burton 03c]. Furthermore, they can detect complete triangulations belonging to a number of infinite families described in [Burton 03b], [Martelli and Petronio 01], and [Matveev 98]. As a result Regina can frequently recognise the underlying 3-manifolds for well-structured triangulations that it has not previously encountered.

2.1.3 Manipulation. For the manipulation of a triangulation, the following procedures are available:

- elementary moves (transformations local to a small number of tetrahedra), such as Pachner moves and other transformations described in [Burton 03c], many of which were suggested by Letscher;

- automated simplification in which the software attempts to use a combination of these elementary moves to reduce the number of tetrahedra as far as possible, though there is no guarantee that the smallest possible number of tetrahedra will be achieved;

- conversion to a 0-efficient triangulation where possible for closed orientable 3-manifolds [Jaco and Rubinstein 02];

- barycentric subdivision and the truncation of ideal vertices (vertices whose links are neither 2-spheres nor discs);

- conversion of a nonorientable triangulation to an orientable double cover; 
- crushing normal surfaces within a triangulation to a point, as described in Section 2.3.2.

\subsection{Census Creation}

Regina can form censuses of all 3-manifold triangulations satisfying various sets of constraints. The census algorithm is described in [Burton 03c] and contains significant optimisations for censuses of closed minimal $\mathbb{P}^{2}$-irreducible triangulations. In particular, the face pairing graph results of [Burton 03a] are incorporated into the algorithm, as are the more standard results relating to low degree edges [Burton 03a, Callahan et al. 99, Matveev 98].

Census creation can require significant amounts of computing time (months or years in some cases). As a result, support is provided for splitting this process into pieces and distributing these pieces amongst several machines.

In addition to forming new censuses, Regina ships with a number of prepackaged censuses including closed 3manifolds [Burton 03b, Burton 03c], cusped hyperbolic 3-manifolds [Callahan et al. 99], and knot and link complements (tabulated by Joe Christy). A census lookup facility for arbitrary triangulations is provided.

\subsection{Normal Surfaces}

The theory of normal surfaces is a powerful tool for the study of 3-manifolds and for the development of algorithms for their analysis. Normal surfaces were introduced by Kneser [Kneser 29] and further developed by Haken [Haken 61, Haken 62], who used them to construct an algorithm for recognising the unknot. Haken furthermore began the construction of an algorithm for solving the homeomorphism problem for a certain large class of 3-manifolds. Difficulties with the methods of Haken were resolved by Jaco and Oertel and by Hemion [Jaco and Oertel 84, Hemion 92], leading to a finite time algorithm for determining whether two closed irreducible 3-manifolds are homeomorphic in the case in which one of these 3-manifolds contains an embedded two-sided incompressible surface.

Normal surfaces feature in a number of 3-manifold decomposition, homeomorphism, and recognition algorithms [Jaco et al. 02, Jaco and Tollefson 95, Rubinstein 95, Rubinstein 97] as well as in algorithms for the simplification of 3-manifold triangulations [Jaco and Rubinstein 02, Jaco and Rubinstein 03a]. For a more extensive review of normal surface theory, the reader is referred to [Hemion 92].
2.3.1 Creation. Providing a computational tool for the study of normal surfaces was in fact the original motivation behind this software. As such, Regina is capable of enumerating all vertex normal surfaces or almost normal surfaces ${ }^{1}$ within a triangulation, an operation required by most high-level topological algorithms that utilise normal surface theory.

Regina can perform this vertex enumeration in a variety of coordinate systems. For an $n$-tetrahedron triangulation this includes the $7 n$ standard triangle and quadrilateral coordinates, as well as the smaller set of $3 n$ quadrilateral-only coordinates introduced by Tollefson for algorithmic efficiency [Tollefson 98]. The enumeration can be restricted to embedded normal surfaces or can be expanded to include immersed and singular surfaces. Furthermore, elementary support is present for spun normal surfaces, which are noncompact surfaces with infinitely many discs found in ideal triangulations [Tillmann 02].

2.3.2 Analysis. For the analysis of normal surfaces, Regina offers the following facilities:

- viewing normal surfaces in a variety of coordinate systems, including the standard and quadrilateralonly coordinates discussed above as well as the edge weight coordinates introduced by Casson;

- calculating basic properties of normal surfaces such as Euler characteristic, orientability and onesidedness;

- recognising standard surfaces within a triangulation such as splitting surfaces (see Section 2.3.3 below) and vertex and edge links;

- filtering large lists of normal surfaces by various properties, such as Euler characteristic, orientability, and boundary.

In addition, the program can crush a normal surface to a point within a triangulation. Crushing is a powerful tool for the analysis of the role played by a surface within a 3-manifold and is used in Jaco and Rubinstein's 0-efficiency algorithm [Jaco and Rubinstein 02].

2.3.3 Splitting Surfaces. Splitting surfaces represent a particular class of normal surfaces whose presence can offer insight into the triangulations containing them. A splitting surface contains precisely one quadrilateral disc

\footnotetext{
${ }^{1}$ Almost normal surfaces are closely related to normal surfaces and are used by Rubinstein in his 3-sphere recognition algorithm [Rubinstein 95, Rubinstein 97].
} 
within each tetrahedron and no other normal or almost normal discs. These surfaces have a number of interesting combinatorial and topological properties, described in detail in [Burton 03b].

As mentioned earlier, Regina can detect whether splitting surfaces occur within a triangulation. It also provides support for splitting surface signatures, which are compact text-based representations from which splitting surfaces and their enclosing 3-manifold triangulations can be reconstructed. In addition to performing such reconstructions, the software can form censuses of all possible splitting surface signatures of a given size.

\subsection{Angle Structures}

Angle structures, studied originally by Casson and then developed by Lackenby and Rivin [Lackenby 00a, Lackenby 00b, Rivin 94, Rivin 03], represent a purely algebraic generalisation of hyperbolic structures. An angle structure on an ideal triangulation is formed by assigning an interior dihedral angle to each edge of every tetrahedron in such a way that a variety of linear equations and inequalities are satisfied.

The formation of angle structures is remarkably similar to the formation of normal surfaces, in which a series of triangle and quadrilateral coordinates are assigned to every tetrahedron with a set of linear equations and inequalities similarly imposed upon them. Thus, it has been relatively straightforward to extend the normal surface enumeration code used by Regina in such a way that the software can also enumerate vertex angle structures.

Included in the requirements of an angle structure is the condition that each dihedral angle $\theta$ satisfies $0 \leq$ $\theta \leq \pi$. In addition to the enumeration of vertex angle structures, Regina can identify whether a triangulation supports any strict angle structures (for which each dihedral angle $\theta$ satisfies $0<\theta<\pi$ ) or any taut angle structures (for which each dihedral angle is precisely 0 or $\pi)$.

\subsection{Scripting}

Regina offers the ability to write and run arbitrary scripts in the Python scripting language. These scripts are essentially high-level programs with immediate access to the mathematical core of Regina and are ideal for performing repetitive tasks over large sets of data. Such tasks might include performing a sequence of tests upon all triangulations in a census, or testing a prototype for a new algorithm. Regina data files can contain embedded scripts, and different files can share code through the use of external libraries of routines.

\subsection{Interfaces and Documentation}

The usual method of running Regina provides a full graphical interface that a user can easily understand and use. Alternatively, for those requiring immediate access to the mathematical core of the software, an interactive command-line interface is offered from which users can control the program using the Python scripting language described above. A variety of specialised utility programs are also available.

Significant effort has been spent on documentation for the software. A full reference manual is available for end users to assist them in working with Regina. This reference manual can be read online at http://regina. sourceforge.net/docs/. For users writing Python scripts or for programmers seeking to modify or extend the software, the routines offered by the underlying mathematical core are also fully documented.

\subsection{Data Files}

The data files used for saving triangulations and other information adhere to a well-organised hierarchical structure. This structure not only allows multiple triangulations, normal surface lists, and other topological structures to be stored together in an organised fashion, but it also supports the storing of miscellaneous data such as text notes and Python scripts. The file format is well documented in the reference manual and uses compressed $\mathrm{XML}^{2}$, allowing for the simple transfer of native Regina data to and from other programs.

\section{APPLICATIONS}

We close with some examples of research projects in which Regina has been used with success.

- In [Burton 03c] a census is presented of all closed nonorientable minimal $\mathbb{P}^{2}$-irreducible triangulations formed from less than or equal to 7 tetrahedra. Computational support from Regina was required not only for the formation of the census but also for the detailed combinatorial analysis of the resulting triangulations. A similar census of orientable triangulations appears in [Burton 03b], again relying upon Regina for much computational support.

- Various constraints upon the structures of minimal triangulations are proven in [Burton 03a]. For this research Regina was used to obtain and process data

\footnotetext{
${ }^{2} \mathrm{XML}$ is the Extensible Markup Language, an open and widely supported text-based data format.
} 
that originally motivated the results, as well as to measure the subsequent improvements to the census algorithm.

- Research into the existence of taut angle structures on ideal triangulations is described in [Burton et al. 03]. Here Regina was used to process large bodies of census data to locate and subsequently analyse triangulations that do not support taut structures.

- For the studies of 0-efficiency and 1-efficiency described in [Jaco and Rubinstein 02] and [Jaco and Rubinstein 03a], Regina has assisted with the construction and analysis of pathological triangulations.

\section{ACKNOWLEDGMENTS}

The author would like to acknowledge the support of the American Institute of Mathematics, the Australian Research Council, and the Grayce B. Kerr Chair at Oklahoma State University.

\section{REFERENCES}

[Burton 03a] Benjamin A. Burton. "Face Pairing Graphs and 3-Manifold Enumeration." To appear in J. Knot Theory Ramifications, July 2003.

[Burton 03b] Benjamin A. Burton. "Minimal Triangulations and Normal Surfaces." PhD diss., University of Melbourne, 2003. Available from World Wide Web (http:// regina.sourceforge.net/).

[Burton 03c] Benjamin A. Burton. "Structures of Small Closed Non-Orientable 3-Manifold Triangulations." Preprint, November 2003.

[Burton et al. 03] Benjamin A. Burton, Ensil Kang, and J. Hyam Rubinstein. "Triangulations of 3-Manifolds III: Taut Structures in Low-Census Manifolds." In preparation, 2003.

[Callahan et al. 99] Patrick J. Callahan, Martin V. Hildebrand, and Jeffrey R. Weeks. "A Census of Cusped Hyperbolic 3-Manifolds." Math. Comp. 68:225 (1999), 321-332.

[Haken 61] Wolfgang Haken. "Theorie der Normalflächen." Acta Math. 105 (1961), 245-375.

[Haken 62] Wolfgang Haken. "Über das Homöomorphieproblem der 3-Mannigfaltigkeiten. I." Math. Z. 80 (1962), 89-120.

[Hemion 92] Geoffrey Hemion. The Classification of Knots and 3-Dimensional Spaces, Oxford Science Publications. Oxford, UK: Oxford University Press, 1992.

[Jaco et al. 02] William Jaco, David Letscher, and J. Hyam Rubinstein. "Algorithms for Essential Surfaces in 3Manifolds." In Topology and Geometry: Commemorating SISTAG, Contemporary Mathematics 314, pp. 107-124. Providence, RI: Amer. Math. Soc., 2002.
[Jaco and Oertel 84] William Jaco and Ulrich Oertel. "An Algorithm to Decide If a 3-Manifold is a Haken Manifold." Topology 23:2 (1984), 195-209.

[Jaco and Rubinstein 02] William Jaco and J. Hyam Rubinstein. "0-Efficient Triangulations of 3-Manifolds." To appear in J. Differential Geom., July 2002.

[Jaco and Rubinstein 03a] William Jaco and J. Hyam Rubinstein. "1-Efficient Triangulations of 3-Manifolds." In preparation, 2003.

[Jaco and Rubinstein 03b] William Jaco and J. Hyam Rubinstein. "Layered Triangulations of Lens Spaces." In preparation, 2003.

[Jaco and Tollefson 95] William Jaco and Jeffrey L. Tollefson. "Algorithms for the Complete Decomposition of a Closed 3-Manifold." Illinois J. Math. 39:3 (1995), 358406.

[Kneser 29] Hellmuth Kneser. "Geschlossene Flächen in dreidimensionalen Mannigfaltigkeiten." Jahresbericht der Deut. Math. Verein. 38 (1929), 248-260.

[Lackenby 00a] Marc Lackenby. "Taut Ideal Triangulations of 3-Manifolds." Geom. Topol. 4 (2000), 369-395 (electronic).

[Lackenby 00b] Marc Lackenby. "Word Hyperbolic Dehn Surgery." Invent. Math. 140:2 (2000), 243-282.

[Martelli and Petronio 01] Bruno Martelli and Carlo Petronio. "Three-Manifolds Having Complexity at Most 9." Experiment. Math. 10:2 (2001), 207-236.

[Matveev 98] Sergei V. Matveev. "Tables of 3-Manifolds up to Complexity 6." Max-Planck-Institut für Mathematik Preprint Series 67 (1998). Available from World Wide Web (http://www.mpim-bonn.mpg.de/html/preprints/ preprints.html).

[Rivin 94] Igor Rivin. "Euclidean Structures on Simplicial Surfaces and Hyperbolic Volume." Ann. of Math. (2) 139:3 (1994), 553-580.

[Rivin 03] Igor Rivin. "Combinatorial Optimization in Geometry." Adv. in Appl. Math. 31:1 (2003), 242-271.

[Rubinstein 95] J. Hyam Rubinstein. "An Algorithm to Recognize the 3-Sphere." In Proceedings of the International Congress of Mathematicians (Zürich, 1994), Vol. 1, pp. 601-611. Basel: Birkhäuser, 1995.

[Rubinstein 97] J. Hyam Rubinstein. "Polyhedral Minimal Surfaces, Heegaard Splittings and Decision Problems for 3-Dimensional Manifolds." In Geometric Topology (Athens, GA, 1993), AMS/IP Stud. Adv. Math., Vol. 2, pp. 1-20. Providence, RI: Amer. Math. Soc., 1997.

[Tillmann 02] Stephan Tillmann. On Character Varieties: Surfaces Associated to Mutation 8 Deformation of Hyperbolic 3-Manifolds." $\mathrm{PhD}$ diss., University of Melbourne, 2002.

[Tollefson 98] Jeffrey L. Tollefson. "Normal Surface QTheory." Pacific J. Math. 183:2 (1998), 359-374. 
[Turaev and Viro 92] Vladimir G. Turaev and Oleg Y. Viro. "State Sum Invariants of 3-Manifolds and Quantum 6jSymbols." Topology 31:4 (1992), 865-902.
[Weeks 91] Jeffrey R. Weeks. "SnapPea: Hyperbolic 3Manifold Software." Available from World Wide Web (http://www.northnet.org/weeks/index/SnapPea. html), 1991-2000.

Benjamin A. Burton, Department of Mathematics and Statistics, The University of Melbourne, VIC 3010, Australia (bab@debian.org)

Received December 9, 2003; accepted February 23, 2004. 